\title{
Continuing Medical Education
}

\section{PATHOPHYSIOLOGY OF TYPE 2 DIABETES}

\author{
A. J. Scheen \\ Key-words : Adipose tissue, Adipocytokins, Insulin resistance, Insulin secretion, Obesity, Type 2 diabetes
}

\begin{abstract}
Type 2 diabetes mellitus is a heterogeneous syndrome characterized by abnormalities in carbohydrate and fat metabolism. The causes of type 2 diabetes are multifactorial and include both genetic and environmental elements that affect beta-cell function and tissue (muscle, liver, adipose tissue, pancreas) insulin sensitivity. Although there is considerable debate as to the relative contributions of beta-cell dysfunction and reduced insulin sensitivity to the pathogenesis of diabetes, it is generally agreed that both these factors play important roles. However, the mechanisms controlling the interplay of these two impairments are unclear. A number of factors have been suggested as possibly linking insulin resistance and beta-cell dysfunction in the pathogenesis of type 2 diabetes. A majority of individuals suffering from type 2 diabetes are obese, with central visceral adiposity. Therefore, the adipose tissue should play a crucial role in the pathogenesis of type 2 diabetes. Although the predominant paradigm used to explain this link is the portal/visceral hypothesis giving a key role in elevated non-esterified fatty acid concentrations, two new emerging paradigms are the ectopic fat storage
\end{abstract}

Division of Diabetes,

Nutrition and Metabolic Disorders,

Department of Medicine,

CHU Sart Tilman,

Liège, Belgium.

Address for correspondence :

A. J. Scheen

Department of medicine

CHU Sart Tilman (b35)

B-4000 Liege 1

Belgium

Phone : $32-4-3667238$

Fax : 32-4-3667068

E-mail : andre.scheen@ chu.ulg.ac.be syndrome (deposition of triglycerides in muscle, liver and pancreatic cells) and the adipose tissue as endocrine organ hypothesis (secretion of various adipocytokins, i.e. leptin, TNF- $\alpha$, resistin, adiponectin, implicated in insulin resistance and possibly beta-cell dysfunction). These two paradigms constitute the framework for the study of the interplay between insulin resistance and beta-cell dysfunction in type 2 diabetes as well as between our obesogenic environment and diabetes risk in the next decade.

Over the last decade, major advances have been made in our understanding of the pathophysiology and molecular biology of type 2 diabetes $(1,2)$. Type 2 diabetes is a bipolar disease characterized by a defect in both insulin secretion and insulin action whose complex interaction leads to a progressive increase of plasma glucose levels (3). It is also well established that the development of type 2 diabetes results from an interaction of a subject's genetic makeup (4) and their environment, and that with the increasing prevalence of obesity, the prevalence of type 2 diabetes is reaching epidemic proportions (5) (Figure 1). Various organs play a crucial role in the pathophysiology of type 2 diabetes. Disruption of the cross-talk between endocrine pancreas, liver, skeletal muscle, adipose tissue and, presumably, gut and central nervous system may lead to alteration of glucose homeostasis and type 2 diabetes (Figure 2) $(6,7)$.

While most patients with type 2 diabetes are overweight or obese (5), the role of fat was initially neglected in the pathophysiology of the disease (6). Its role was highlighted almost a decade ago, especially the interactions of non-esterified fatty acids (NEFA) with glucose metabolism (7). The crucial impact of fat distribution, especially the negative influence of intra-abdominal or visceral fat depot, is now largely recognized (8). More recently, the deleterious role of ectopic triglyceride storage in the development of defective insulin action and insulin secretion has been emphasized leading to 


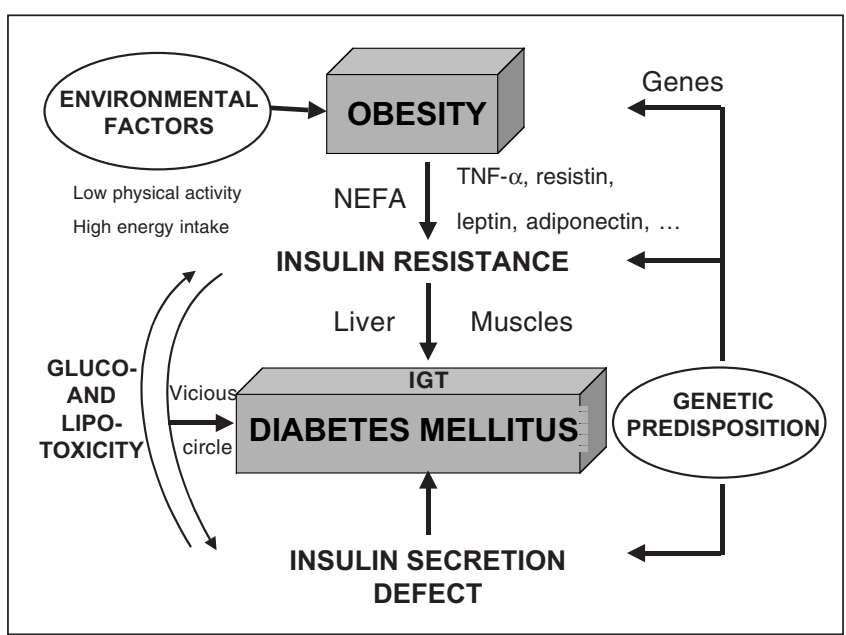

Figure 1 : Contribution of genetic predisposition and environment factors in the pathogenesis of type 2 diabetes and interplay between defective insulin secretion and insulin resistance leading to a vicious circle explaining the progression from impaired glucose tolerance (IGT) to type 2 diabetes and the progressive aggravation of the disease.

the concept of lipotoxicity (9). Finally, as discussed at the end of this review, adipose tissue can secrete various molecules that may interfere with glucose metabolism and insulin sensitivity, such as leptin, tumour necrosis factor (TNF)- $\alpha$, resistin, adiponectin, ... (10).

While it is clear that hyperglycaemia is associated with both insulin resistance and beta-cell dysfunction, there has been much debate over the past few decades regarding the relative importance and sequence of these two abnormalities. This disagreement over the relative importance of these two defects in type 2 diabetes is partly due to the fact that evaluation of insulin resistance and beta-cell dysfunction has frequently been done in isolation, thus not taking into account the fact that glucose homeostasis is vitally dependent on a feedback system (3). Once consideration is given to the presence of a tightly regulated feedback system incorporating the beta cell and the insulin-sensitive tissues (hyperbolic relationship between insulin secretion and insulin sensitivity), it becomes abundantly clear that reductions in both insulin sensitivity and beta-cell function are present early in the course of the development of type 2 diabetes (3).

\section{ROLE OF INSULIN RESISTANCE IN TYPE 2 DIABETES}

Even if insulin exerts numerous different effects, so far insulin sensitivity has been considered mainly in the context of glucose metabolism, especially at the liver and muscle sites (11). The presence of insulin resistance in vivo can be evidenced during various dynamic tests such as an oral glucose tolerance test, an intravenous glucose tolerance test and a so-called euglycaemic hyperinsulinaemic clamp (12). Using the latter approach, it has been extensively demonstrated that insulin-mediated glucose disposal (essentially in the skeletal muscle) is largely reduced in patients with type 2 diabetes. Furthermore, the concomitant use of isotopes showed that hepatic glucose production is insufficiently inhibited by insulin, thus demonstrating the presence of both muscular and hepatic insulin resistance.

Despite tremendous advances in molecular biology and the continued identification of increasingly more molecules involved in the insulin signaling cascade, the molecular mechanism (or mechanisms) that underlines the development of insulin resistance in subjects prone to develop type 2 diabetes still remains elusive (4). Genetic mutations associated with insulin resistance are rare and it seems unlikely that a single genetic alteration explains a large number of cases of insulin resistance among type 2 diabetic patients. Rather, it is more likely that a number of different genes may contribute, some of which may be obesity genes. Three commonly encountered factors that influence insulin sensitivity and are apparently not genetically determined are aging, exercise and dietary constituents. However, even if the effects of age, exercise and diet are considered, there is still a large between-subject variation in insulin sensitivity that has to be related to other factors. A major component of this residual va-

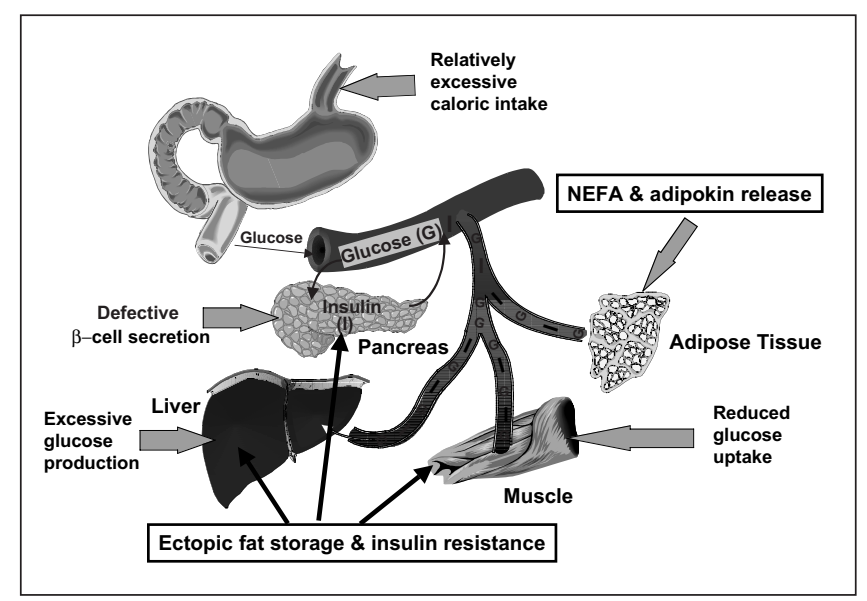

Figure 2 : Contribution of endocrine pancreas, liver, skeletal muscle and adipose tissue in the pathogenesis of type 2 diabetes : emerging role of ectopic fat storage in liver, muscle and beta-cell and of adipose tissue as an endocrine organ releasing various adipocytokins in addition to non-esterified fatty acids (NEFA) in presence of positive energy balance and obesity. . 
riation may be related to obesity, but more importantly to differences in body fat distribution (8).

A vast majority of type 2 diabetic patients are overweight, and obesity undoubtedly plays a major role in the development of the disease $(1-3,5)$. While it is recognized that obesity is an important determinant of insulin sensitivity (12), body-fat distribution seems to be a critical aspect (8). Several groups have made a strong case that the intra-abdominally fat depot is the primary correlate of insulin sensitivity, while others have proposed that the central subcutaneous fat depot is the major factor determining a reduction in insulin sensitivity. Obese individuals with most of their fat stored in visceral adipose depots generally suffer greater adverse metabolic consequences than similarly overweight subjects with fat stored predominantly in subcutaneous sites. Excess abdominal fat mass is associated with an increased release of NEFA that may trigger a reduction in insulin sensitivity at both the hepatic and the muscular levels. In the liver, this results in an increased glucose output (essentially due to enhanced gluconeogenesis), a decreased insulin extraction and an increased VLDL production while in the skeletal muscle this results in a reduction in glucose oxidation and glucose storage as glycogen (so-called Randle's effect) $(1,7)$. Numerous insulin-resistant obese patients have also a so-called metabolic syndrome associating impaired glucose tolerance (or type 2 diabetes), dyslipidaemia and arterial hypertension, all factors aggravating the risk of cardiovascular diseases (13). A fuller understanding of the biology of central obesity will require information regarding the genetic and environmental determinants of human fat topography and of the molecular mechanisms linking visceral adiposity to degenerative metabolic and vascular disease.

Long-term positive energy balance may lead not only to excess triglyceride depots in the adipose tissue, but also to ectopic triglyceride storage. The ability of the adipocyte to function properly when engorged with lipid can lead to lipid accumulation in other tissues, reducing their ability to function and response normally. Liver steatosis is a common finding in obese subjects, especially in those with intra-abdominal fat depot, and non-alcoholic fatty liver disease is now considered as part of the metabolic syndrome associated to insulin resistance. In addition, intramuscular triglyceride levels are increased in obese subjects, and a close relationship has been repeatedly reported between the degree of ectopic intramyocellular triglyceride depot and the severity of muscular insulin resistance (14). Interestingly, ectopic fat accumulation in insulin-sensitive tissues may be associated with insulin resistance independent of overall obesity. However, the understanding of the causes and mechanisms underlying fat accumulation in skeletal muscle and the liver are limited. Identifying why some individuals store fat in insulin-sensitive tissues, but others do not, may be of great importance for the development of new insulinsensitizing agents.

The role of counterregulatory hormones in the resistance to insulin in patients with type 2 diabetes remains unclear. Nevertheless, plasma glucagon levels are regularly increased in type 2 diabetic patients and this hormone could contribute to enhance gluconeogenesis and hepatic glucose output, especially in presence of insulin deficiency (1). Pharmacological attempts to decrease glucagon secretion led to substantial reduction in plasma glucose levels, arguing for a significant role of this hormone in the development of hyperglycaemia in type 2 diabetes.

Finally, an haemodynamic hypothesis of insulin resistance has also been put forward (1). Reduced number of muscle capillaries and impaired insulin-induced vasodilatation (essentially in the postprandial state) may contribute to increase the distance and to alter the insulin diffusion process from the capillary to the muscular cells and thereby insulin action in obese patients with type 2 diabetes (1). This circulatory phenomenon may explain recent consistent findings showing that inhibition of the renin-angiotensin system (with either angiotensin converting enzyme inhibitors or AT1 receptor antagonists) is able to reduce the incidence of type 2 diabetes in hypertensive insulin-resistant patients.

\section{ROLE OF INSULIN DEFICIENCY IN TYPE 2 DIABETES}

Beta-cell function in type 2 diabetes has been the subject of intense investigation for several decades, and considerable progress has been made during the recent years in the knowledge of the physiology and pathophysiology of insulin secretion (15). Recent data demonstrated that beta-cell deficit and beta-cell apoptosis are present in humans with type 2 diabetes (16). Given the number of variables that could contribute to a disordered insulin secretory response, it is obvious that a precise definition of the sequence of events that leads to this disorder in a given patient with common type 2 diabetes remains, in most cases, an elusive goal. 
Once hyperglycaemia exists, beta-cell dysfunction is clearly present in subjects with type 2 diabetes. This change manifests in a number of different ways including decreases in the early insulin response to intravenous or oral glucose and a decline in the ability of glucose to potentiate the insulin response to non-glucose secretagogues (15). Individuals with type 2 diabetes show also a decease in the potentiation by oral rather than parenteral glucose loading, a phenomenon known as the "incretin effect" which is associated to glucose-dependent insulinotropic peptide (GIP) and glucagon-like peptide (GLP)-1 secreted by enterocytes. In addition, alterations in pulsatile insulin release and ultradian oscillatory insulin secretion can be observed. Finally, inefficient proinsulin processing to insulin and a reduction in the release of islet amyloid polypeptide (IAPP, also known as amylin) have been observed in established type 2 diabetes (3).

Recent examination of a number of different parameters in various human models has emphasised the fact that beta-cell dysfunction, when assessed using appropriate methods, is present well before the onset of hyperglycaemia, i.e. in subjects with impaired glucose tolerance and even in first-degree relatives with normal glucose tolerance. First-phase insulin response to glucose is reduced in an early stage, thus contributing to postprandial hyperglycaemia, while late-phase insulin secretion may be preserved for a longer term as it is triggered by sustained hyperglycaemia. A backward extrapolation of findings made in the United Kingdom Prospective Diabetes Study (UKPDS) cohort from shortly after the clinical diagnosis of type 2 diabetes strongly suggests that beta-cell dysfunction commences years (at least 10 years) before hyperglycaemia develops (17).

Three main mechanisms have been proposed to explain the B-cell deficiency observed in subjects prone to develop type 2 diabetes (1). First, a genetic defect may be present, although such a defect has not been detected yet in subjects with common type 2 diabetes associated with obesity, in contrast to what was reported in some particular forms of "Maturity-Onset Diabetes of the Youth" (MODY) (mutation of the glucokinase gene in MODY 3 , for instance) (15). Second, in utero malnutrition may lead to insufficient beta-cell development and later partial insulin secretory defect. This hypothesis has been called the "thrifty phenotype hypothesis" (18). However, the relationship with insulin secretion seems to be less clear than the relationship with insulin resistance and the metabolic syndrome. And third, unfavourable metabolic environment may also play a deleterious role, especially increased glucose levels that may induce glucotoxicity (19) and a chronic increase in NEFA levels that may induce lipotoxicity (9), both processes contributing to alter insulin secretion. Interestingly, ectopic deposition of triglycerides in pancreatic islets has also been reported, a condition that may contribute to dysfunction of the beta cell (9). Indeed, although the mechanism of lipotoxicity in the beta cell remains unclear, it has been suggested that accumulation of triglycerides increases nitric oxide, which causes oxidative damage and apoptosis in the cells (9). Finally, defects in insulin signalling pathways associated with insulin resistance in peripheral tissues have recently been found to disrupt insulin secretion by pancreatic beta cells, suggesting that insulin resistance in the beta cells may be, at least partly, responsible for the beta-cell dysfunction and the development of type 2 diabetes (10).

\section{DYNAMIC INTERACTION BETWEEN INSULIN ACTION AND INSULIN SECRETION}

Subjects with type 2 diabetes are characterized by both tissue insulin resistance and impaired insulin secretion $(3,20)$. The development of diabetes requires the presence of these two fundamental defects, which disrupt the delicate balance by which insulin-target tissues communicate with the beta cells and vice versa. Numerous observations underscore the important interplay between insulin resistance and insulin secretion $(1,3)$. Both abnormalities must be looked at in concert and the relative importance of these two factors can be estimated with the aid of a theoretical mathematical model of glucose metabolism. Insulin resistance alone is, in most instances, insufficient to cause overt glucose intolerance. To observe the development of frank diabetes mellitus, a defect in insulin secretion must be superimposed on insulin resistance $(1,3)$, and numerous observations suggest that this beta-cell defect might be genetically determined (21).

Type 2 diabetes occurs as a late phenomenon in obese subjects and is preceded by years of normal glucose tolerance or impaired glucose tolerance (IGT) as suggested by several cross-sectional studies $(1,3,8)$. The progression from IGT to diabetes occurs when the beta cell becomes unable to maintain its previously high rate of insulin secretion in response to glucose (22). Longitudinal studies confirmed that this evolution occurs 
along the natural history of obesity $(8,22)$. During the first years of obesity, the subjects are normoglycaemic but hyperinsulinaemic. Afterwards they become hyperglycaemic at a time when hyperinsulinaemia is not maintained anymore. To some extent, the natural history of the obese subjects developing type 2 diabetes after a prolonged phase of compensatory hyperinsulinism may be explained by the "overworked beta cell" hypothesis, an hypothesis supported by observations in various animal models (23).

Once it develops, either from insulin deficiency or from insulin resistance, hyperglycaemia will exacerbate both defects, thereby closing a pathological feedback loop (Figure 1). Recent data from the UKPDS demonstrated that in newly diagnosed type 2 diabetes, the progressive deterioration of blood glucose control over the next 10 years can essentially be explained by a linear decrease of beta-cell insulin secretory capability (17). Even though our understanding of the mechanisms leading to the appearance and the progression of type 2 diabetes is incomplete, the counterproductive interplay between insulin resistance and insulin deficiency, and the concepts of glucose (19) and lipid (9) toxicity have important clinical and therapeutical implications (24). In the scenario of "glucose toxicity" or "glucose desensitization" (19), glucose lowering is associated with improvements in both insulin action and insulin release. In the scenario of lipotoxicity (9), reduction in NEFA concentrations and ectopic fat storage after sustained negative energy balance and weight loss may also result in both enhanced insulin sensitivity and insulin secretion $(8,22)$. Consequently, aggressive treatment targeted to even modest hyperglycaemia and weight excess should be recommended in order to slow down the development and progression of type 2 diabetes.

While most investigators agree that insulin secretion and insulin action must both be impaired for the development of overt diabetes, there is no consensus as to which comes first $(3,20)$. Owing to increasing evidence of heterogeneity of the disease, it is plausible that each defect will prove to be primary in some forms of type 2 diabetes. The answer will most certainly be provided by genetic analysis. Indeed, genetic research of type 2 diabetes is undergoing rapid development, and many studies are in progress, especially using genome-wide scans which may lead to new genes. However, despite exciting developments, the genetic susceptibility of common type 2 diabetes still remains an enigma.

\section{ADIPOSE TISSUE AS AN ENDOCRINE ORGAN}

The view of the adipocyte as a simply storage depot for fat is no longer tenable. Indeed, the role of adipose tissue as an endocrine organ capable of secreting a number of adipose- tissue-specific or enriched hormones, known as adipocytokines or adipokines, is gaining appreciation. Indeed, besides NEFA, adipocytes secrete various cytokines, among which leptin, TNF- $\alpha$, resistin and adiponectin $(9,10,14)$. The role of leptin in carbohydrate metabolism remains unclear whereas TNF- $\alpha$ and resistin clearly inhibit cellular insulin action (10). However, most studies were performed in rodents and the contribution of these two cytokines to hyperglycaemia of type 2 diabetes is still controversial in humans. Among the various adipocytokines, adiponectin appears to play a very important role in carbohydrate and lipid metabolism (25). Indeed, adiponectin, an abundant circulating protein synthesized solely in adipose tissue, appears to be a major modulator of insulin action. In contrast to other adipocytokines, adiponectin is characterized by lower (and not higher) circulating levels in presence of obesity. In addition, whereas leptin is more positively related to subcutaneous than to intra-abdominal fat, adiponectin is more strongly negatively related to intra-abdominal than to subcutaneous fat. Its levels are reduced in type 2 diabetes and a strong positive relationship between insulin sensitivity and adiponectin levels has been described in various populations. Thus, low adiponectin levels could contribute to peripheral insulin resistance in type 2 diabetes. The mechanisms by which adiponectin is synthesized need to be elucidated, as do the signals that reduce adiponectin expression in adipocytes with increasing adiposity. Similarly, the molecular mechanisms by which adiponectin exerts its multiple functions still remain a mystery as well as its potential role in the development of type 2 diabetes in obese subjects, especially those with intra-abdominal adiposity. Although further investigations are required, adiponectin administration, as well as regulation of the pathways controlling its production (new insulin sensitizers thiazolidinediones, also called glitazones, have been shown to increase adiponectin concentrations) represents a promising target for managing insulin resistance and type 2 diabetes (25). 


\section{CONCLUSIONS}

The development of type 2 diabetes requires the presence of two fundamental defects, i.e. insulin resistance and impaired insulin secretion, which disrupt the delicate balance by which insulin target tissues communicate with the beta cells and vice versa. Thus, type 2 diabetes is a heterogeneous disorder characterized by impaired insulin secretion, diminished peripheral (muscular) insulin action, and increased hepatic glucose production, all defects being present in variable proportions in different individuals, but also prone to changes in a given individual with the progression of the disease. Recent data from UKPDS and other studies clearly showed that the progression of hyperglycaemia characterizing the natural history of type 2 diabetes essentially results from an aggravation of beta-cell defect. Thus, besides efforts to correct insulin resistance, preserving beta-cell function represents a key-objective for the future management of type 2 diabetes (26).

Obesity, especially intra-abdominal adiposity, plays a crucial role in the development of the disease. Numerous mechanisms have been proposed including elevated NEFA circulating levels, ectopic fat storage exerting a toxic effect in liver, muscle and pancreas, and secretion of various adipocytokins that may interfere with insulin action and possibly insulin secretion. In addition, all the abnormalities linked to fat tissue are intimately associated with the so-called metabolic syndrome frequently observed in obese individuals with type 2 diabetes who are at high risk of cardiovascular diseases.

Even though we are not yet able to pinpoint the primary defect in most patients with type 2 diabetes, there appears to be a counterproductive interplay between beta-cell inadequacy and insulin resistance, which leads to a vicious circle that perpetuates and aggravates the metabolic disorder. Based on our greater understanding of the relative roles of insulin resistance and beta-cell dysfunction in type 2 diabetes and the increasingly recognized role of adipose tissue and ectopic fat storage, we can anticipate advances in the identification of genes contributing to the development of the disease as well as approaches to the treatment and prevention of type 2 diabetes.

\section{REFERENCES}

1. Scheen AJ, Lefèbvre PJ. Pathophysiology of Type 2 diabetes. In: Kuhlmann J, Puls W, eds. Handbook of Experimental Pharmacology, Oral Antidiabetics. Berlin: Springer Verlag, 1996: 7-42.

2. DeFronzo RA. Pathogenesis of type 2 diabetes : metabolic and molecular implications for identifying diabetes genes. Diabetes Rev 1997; 5: 177-269.

3. Kahn SE. The relative contributions of insulin resistance and beta-cell dysfunction to the pathophysiology of Type 2 diabetes. Diabetologia 2003; 46: 3-19.

4. Gerich J. The genetic basis of type 2 diabetes mellitus : impaired insulin secretion versus impaired insulin sensitivity. Endocr Rev 1998; 19: 491-503.

5. Scheen AJ. Obesity and diabetes. In : The management of obesity and related disorders (Kopelman P.G., Ed.), Martin Dunitz Ltd, London, UK, 2001: 11-44.

6. DeFronzo RA. The triumvirate : B-cell, muscle, liver. A collusion responsible for NIDDM. Diabetes 1988; 37: 667-87.

7. Reaven GM. The fourth Musketeer - from Alexandre Dumas to Claude Bernard. Diabetologia 1995; 38: 3-13.

8. Montague CT, O'Rahilly S. The perils of portliness. Causes and consequences of visceral adiposity. Diabetes 2000; 49: 883-8.

9. Unger RH. Lipotoxic diseases. Annu Rev Med 2002; 53: 31936.

10. Greenberg AS, McDaniel ML. Identifying the links between obesity, insulin resistance and $\beta$-cell function : potential role of adipocyte-derived cytokines in the pathogenesis of type 2 diabetes. Eur J Clin Invest 2002; 32 (Suppl 3): 24-34.

11. Scheen AJ, Lefèbvre PJ. Assessment of insulin resistance in vivo. Application to the study of type 2 diabetes. Horm Res 1992; 38: 19-27.

12. Scheen AJ, Paquot N, Letiexhe MR, Paolisso G, Castillo MJ, Lefèbvre PJ. Glucose metabolism in obese subjects : lessons from OGTT, IVGTT and clamp studies. Int J Obesity 1995; 19 (Suppl 3): S14-S20.

13. Scheen AJ. Insulin resistance syndrome and atherosclerotic cardiovascular disease. Acta Clin Belg 1996; 51: 65-9.

14. Ravussin E, Smith SR. Increased fat intake, impaired fat oxidation, and failure of fat cell proliferation result in ectopic fat storage, insulin resistance, and type 2 diabetes mellitus. Ann $N$ Y Acad Sci 2002; 967: 363-78.

15. Polonsky KS. The beta-cell in diabetes : from molecular genetics to clinical research. Diabetes 1995; 44: 705-17.

16. Butler AE, Janson J, Bonner-Weir S, Ritzel R, Rizza RA, Butler PC. $\beta$-cell deficit and increased $\beta$-cell apoptosis in humans with type 2 diabetes. Diabetes 2003; 52: 102-10.

17. UKPDS Group. UK Prospective Diabetes Study 16: overview of six years' therapy of type 2 diabetes - a progressive disease. Diabetes 1995; 44: 1249-58.

18. Hales CN, Ozanne SE. Fetal and early postnatal growth restriction lead to diabetes, the metabolic syndrome and renal failure. Diabetologia 2003; 46: 1013-9.

19. Yki-Jarvinen H. Glucose toxicity. Endocr Rev 1992; 13: 41531 .

20. Scheen AJ, Lefèbvre PJ. Insulin resistance vs. insulin deficiency : which comes first? The old question revisited. In : Diabetes in the New Millennium ( Eds : Di Mario U., Leonetti F., Pugliese G., Sbraccia P. and Signore A.), J. Wiley \& Sons, New York, 2000: 101-13. 
21. Polonsky KS, Sturis J, Bell GI. Non-insulin-dependent diabetes mellitus : a genetically programmed failure of the beta cell to compensate for insulin resistance. N Engl J Med 1996; 334: 777-83.

22. Scheen AJ. From obesity to diabetes. Why, when and who ? Acta Clin Belg 2000; 55: 9-15.

23. Leahy JL. Impaired ß-cell function with chronic hyperglycemia : “overworked B-cell” hypothesis. Diabetes Rev 1996; 4: 298319.
24. Saltiel AR. New perspectives into the molecular pathogenesis and treatment of type 2 diabetes. Cell 2001; 104: 517-29.

25. Chandran M, Phillips SA, Ciaraldi T, Henry RR. Adiponectin : more than just another fat cell hormone? Diabetes Care 2003; 26: 2442-50.

26. Scheen A. J. Treatment of type 2 diabetes. Acta Clin Belg 2003; 58: 318-24. 